\title{
The marker level of endogenous intoxication in the midst of bronchopneumonia and during the convalescence of calves
}

\author{
Yurij Alekhin ${ }^{1 *}$, Maksim Zhukov ${ }^{1}$, S.A. Grin ${ }^{2}$, and A.G. Koshchaev ${ }^{3}$ \\ ${ }^{1}$ All-Russian Veterinary Research Institute of Pathology, Pharmacology and Therapy, 394087 Voronezh, Russia \\ ${ }^{2}$ All-Russian Research and Technology Institute of Biological Industry, 107140 Moscow, Russia \\ ${ }^{3}$ Kuban State Agrarian University named after I. T. Trubilin, 350044 Krasnodar, Russia
}

\begin{abstract}
The research on the level of markers of endogenous intoxication in the midst of bronchopneumonia and during convalescence in calves was conducted in conditions of a complex for feeding young cattle. The experiment involved calves aged 4-5 months, group $1-$ clinically healthy $(\mathrm{n}=$ $30)$, group 2 - animals with bronchopneumonia with moderate severity of the disease $(n=72)$ subjected to full treatment. Blood sampling was carried out the day the groups were formed (in calves from group 2, this is the period of "high disease"), as well as on days corresponding to days $1,3,6,10,13,18$, and 25 after the disappearance of specific symptoms of bronchopneumonia. From the markers of endogenous intoxication syndrome in the blood, the content of medium-weight molecules (AWM), the sorption capacity of erythrocytes, non-erythrocyte hemoglobin by the hemoglobin cyanide method, and malondialdehyde were determined. Studies have shown that in $37.5 \%$ of those who have suffered, intoxication markers disappear within 10-13 days after completing the course of treatment, and in $41.7 \%$ of calves after positive dynamics, the AWM level increases at 10-13 days at a wavelength of $237 \mathrm{~nm}$, which indicates activation of the resorptive mechanism of autointoxication and the risk of relapse. At the same time, the dynamics of recovery processes in $20.8 \%$ of patients who were ill was characterized by normalization of most of the studied parameters, but by maintaining an increased level of lipid peroxidation products and transmembrane hemoglobin loss, which can cause secondary anemia and re-illness.
\end{abstract}

\section{Introduction}

The issues of obtaining and maintaining healthy young farm animals become one of the most urgent problem in livestock farming determined by its effectiveness. An important role in the the nosological profile formation of calf diseases depends on the respiratory pathology $[1,2]$. Most researchers predict a further increase in the relevance of these diseases, the expansion of the etiological spectrum and a decrease in the effectiveness of control measures [3-5], which indicates the need to find new approaches to solving the problem.

Previous data we obtained showed a high level of incidence of respiratory organs of calves formed because of repeated pain arising due to the presence of residual pathological phenomena [6]. An integral component of the pathogenesis of bronchopulmonary diseases is endogenous intoxication [7]. At a high level of which the possibilities of natural detoxification mechanisms, including the monooxygenase detoxifying system of the liver and excretory system, are significantly limited due to the breakdown of compensatory adaptive mechanisms $[8,9]$, which is likely to disrupt the healing processes with an increase in the severity of residual events and the risk of recurrence of the disease. Therefore, endogenous intoxication is assumed to be one of the residual pathological phenomena in patients with respiratory illnesses and as well as one of the reasons for their repeated illness. In this regard, the purpose of the research is to study markers of endogenous intoxication in bronchopneumonia in calves during the period of convalescence.

\section{Materials and research methods}

This study was approved by the ethics committee of the Federal State Budgetary Scientific Institution " AllRussian Research Veterinary Institute of Pathology, Pharmacology and Therapy of the Russian Academy of Agricultural Sciences". The study was conducted by following the fair treatment of animals. The studies were carried out in a complex for feeding young cattle. In the experiment, calves aged 4-5 months were involved from among whom, on the basis of the principle of selection of analogues, group 1 was clinically healthy (control, $\mathrm{n}=$ 30 ), group 2 - animals with bronchopneumonia with moderate disease severity $(n=72)$, where gram-positive and gram-negative bacteria played a leading role (ICD10: J18.0 - "Unspecified bronchopneumonia"). The diagnosis was made on the basis of clinical, instrumental, bacteriological and serological studies. During the experiment, the animals were kept in a room

Corresponding author: exterapi@yandex.ru 
with comfortable microclimate parameters and were housed in group machines for 12-15 goals. Their diet was complete and consistent with existing feeding standards. Patients were prescribed complex treatment, which included a course of antibiotics (1-7 days), selected on the basis of an assessment of the sensitivity of pathogens (gentamicin sulfate, enrofloxacin), intravenous administration of calcium chloride and glucose solutions ( 1 and 3 days), intramuscular injections of a vitamin preparation "Tetravitam" ( 1 and 7 days). In addition, on 2 (on the left side) and 4 (on the right side) day, novocaine blockade of stellate sympathetic nodes was performed. 24 hours after completion of the course of treatment, a clinical examination of the animals was carried out, the results of which showed that all calves had specific symptoms of bronchopneumonia disappeared. However, markers of endogenous intoxication remained in the blood, according to the level of which three subgroups of animals were distinguished: $2 \mathrm{a}(\mathrm{n}=27), 2 \mathrm{~b}(\mathrm{n}=30)$ and $2 \mathrm{c}(\mathrm{n}=15)$.

All animals in the experiment were under constant clinical supervision, but a more detailed examination and blood sampling was carried out the day groups were formed (in calves from group 2, this is the "peak of illness" period), as well as days corresponding to 1, 3, 6, $10,13,18$ and 25 days after completion of treatment for patients from the second group.

Blood samples were taken from the yarmine vein into tubes with an anticoagulant $(3.8 \%$ sodium citrate solution) to maintain its intact state and with a $\mathrm{SiO} 2$ coagulation activator (Guangzhou Improve Medical Instruments CO., LTD, China) to obtain blood serum. From the markers of endogenous intoxication syndrome in the blood there were determined: 1the content of medium-weight molecules, the sorption capacity (capacity) of erythrocytes [10], non-erythrocyte hemoglobin by the hemoglobin cyanide method [11] and malondialdehyde [12]

Mathematical and statistical processing of the obtained data was performed using the SPSS version 22 program (IBM Corp, Version 22.0, Armonk, NY, USA, 2013). There were arithmetic mean, the average error of the arithmetic calculated, the difference between the experimental groups was evaluated by Student's criterion.

\section{Results}

The studies has shown (Table 1) following results: in animals from group 1 (control), the level of endogenous intoxication markers did not change significantly during the entire observation period. In comparison, the calves from group 2, during the height of the disease, had a significant increase of erythrocyte sorption capacity (capacity) (SCE), 4.6-fold extra-erythrocyte hemoglobin (NEH), and 2.8-fold malondialdehyde was detected by $21.8 \%$ (MDA). The content of medium-mass molecules (AWM) determined at wavelengths of 237, 254 and $280 \mathrm{~nm}$ was 3.4 times higher than the control, respectively, by 37.3 and $52.2 \%$.
Table 1. The level of markers of endogenous intoxication in healthy calves and patients with bronchopneumonia (stage of the height of the disease).

\begin{tabular}{|c|c|c|}
\hline Indicators & $\begin{array}{c}\text { Group 1 } \\
\text { (healthy) }\end{array}$ & $\begin{array}{c}\text { Group 2 } \\
\text { (sick) }\end{array}$ \\
\hline NEH, g / 1 & $0.42 \pm 0.050$ & $1.95 \pm 0.030^{*}$ \\
\hline $\begin{array}{c}\text { AWM 237 nm, } \\
\text { conventional units }\end{array}$ & $0.585 \pm 0.0306$ & $1.980 \pm 0.0226^{*}$ \\
\hline $\begin{array}{c}\text { AWM 254 nm, } \\
\text { conventional units }\end{array}$ & $0.284 \pm 0.0092$ & $0.390 \pm 0.0077^{*}$ \\
\hline $\begin{array}{c}\text { AWM 280 nm, } \\
\text { conventional units }\end{array}$ & $0.247 \pm 0.0058$ & $0.376 \pm 0.0100^{*}$ \\
\hline SCE, \% & $35.7 \pm 0.84$ & $43.5 \pm 1.01^{*}$ \\
\hline MDA, $\mu \mathrm{M} / \mathrm{L}$ & $0.58 \pm 0.023$ & $1.62 \pm 0.017^{*}$ \\
\hline
\end{tabular}

Note $:{ }^{*}-\mathrm{p} \leq 0.001$ in comparison with clinically healthy animals

All animals from group 2 remained elevated levels of endotoxicosis markers the first day after treatment completion. However, the next three days, 27 (37.5\%) calves (subgroup 2a) lost most of the signs of autotoxicity (Table 2). The exception was the concentration of NEH and MDA, which normalized only the 10th day of the postclinical period (Table 2).

In $30(41.7 \%)$ animals (2b), during the entire observation period, the disturbed structure of the erythrocyte membranes did not recover, as shown by the increased indicators of extra-erythrocyte hemoglobin and the sorption capacity of red blood cells. The 25 th day of convalescence, SCE level decreased to the upper limit of the reference interval $(38.5 \%)$.

The content of medium-weight molecules, determined at a wavelength of $237 \mathrm{~nm}$, was in the range of healthy animals, but increased by $32.6 \%$ on day 10 . And although, over the next 3 days, their level decreased by $21.9 \%$, it remained elevated until the end of the experiment.

For the first 13 days of convalescence, AWM values at wavelengths of 254 and $280 \mathrm{~nm}$ were higher than normal (to 0.300 srvc units), but then their values returned to normal. This fact indicates an increase in the usefulness of metabolic processes. Although the activity of lipid peroxidation remained elevated, as evidenced by the concentration of MDA, which during the entire observation period exceeded 2.5-3 times the value of healthy calves.

In $15(20.8 \%)$ animals (2c), the AWM content did not exceed the control level, which indicates the absence of pronounced metabolic disorders, although increased MDA indicates a high activity of lipid peroxidation, which is probably the reason for the preservation of intensive transmembrane excretion hemoglobin. Moreover, despite the noted preservation of structural disorders of the erythrocyte membranes, their functions are restored, as indicated by the normalization of their sorption ability on the 10th day of reconvalescence. 
Table 2. The level of markers of endogenous intoxication in calves during the period of bronchopneumonia convalescence $(\mathrm{M} \pm \mathrm{m})$

\begin{tabular}{|c|c|c|c|c|c|c|c|c|}
\hline \multirow[t]{2}{*}{ Indicators } & \multirow[t]{2}{*}{ Group } & \multicolumn{7}{|c|}{ Convalescence period (days) } \\
\hline & & 1 & 3 & 6 & 10 & 13 & 18 & 25 \\
\hline \multirow[t]{4}{*}{$\mathrm{NEH}, \mathrm{g} / \mathrm{l}$} & 1 & $\overline{45 \pm 0}$ & $2 \pm 0$ & $2 \pm 0$ & $15 \pm$ & $41 \pm$ & $35 \pm 0$ & - \\
\hline & $2 \mathrm{a}$ & $\partial^{3}$ & $3 \pm 0$. & $0.65 \pm 0.029^{3}$ & & 0 & 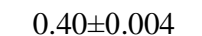 & ? \\
\hline & 20 & $41 \pm 0.012^{3}$ & $0.79 \pm 0.006^{3}$ & $0.80 \pm 0.005^{3}$ & $0.92 \pm 0.006^{3}$ & $0.85 \pm 0.030^{3}$ & $.80 \pm 0.005^{3}$ & $0.86 \pm 0.006^{3}$ \\
\hline & $2 \mathrm{c}$ & $1.34 \pm 0.0$ & $0.73 \pm 0.023^{3}$ & $1.0 \pm 0.023^{3}$ & $1.25 \pm 0.068^{3}$ & $0.67 \pm 0.009^{3}$ & $0.99 \pm 0.004^{3}$ & $.77 \pm 0.005^{3}$ \\
\hline \multirow{4}{*}{$\begin{array}{c}\text { AWM } 237 \\
\text { nm, } \\
\text { conventional } \\
\text { units } \\
\end{array}$} & 1 & $559 \pm 0 .($ & $0.575 \pm 0$. & $0.560 \pm 0.0313$ & $0.563 \pm 0.0300$ & $0.559 \pm 0$ & $0.590 \pm 0.0107$ & $0.568 \pm 0$ \\
\hline & $2 \mathrm{a}$ & $35 \pm 0.0277^{3}$ & $.926 \pm 0.0199^{3}$ & $0.909 \pm 0.0241^{3}$ & $0.601 \pm 0$ & $0.786 \pm 0.0330^{3}$ & $0.796 \pm 0.0165^{3}$ & $0.680 \pm 0.0137^{3}$ \\
\hline & $2 b$ & $1.046 \pm 0.0072^{3}$ & $0.837 \pm 0.0801^{2}$ & $0.980 \pm 0.0106^{3}$ & $1.300 \pm 0.0073^{3}$ & $1.280 \pm 0.0084^{3}$ & $1.000 \pm 0.0117^{3}$ & $1.003 \pm 0.0060^{3}$ \\
\hline & $2 c$ & $1.046 \pm 0.0295^{3}$ & $0.979 \pm 0.0239^{3}$ & $0.850 \pm 0.0116^{3}$ & $0.885 \pm 0.0111^{3}$ & $0.995 \pm 0.0310^{3}$ & $0.950 \pm 0.0182^{3}$ & $0.819 \pm 0.0296^{3}$ \\
\hline \multirow{4}{*}{$\begin{array}{c}\text { AWM } 254 \\
\text { nm, } \\
\text { conventional } \\
\text { units } \\
\end{array}$} & 1 & $0.269 \pm 0.0095$ & $0.257 \pm 0.0073$ & $0.269 \pm 0.0075$ & $0.269 \pm 0.0090$ & 0.2 & 63 & $0.260 \pm 0.0071$ \\
\hline & $2 \mathrm{a}$ & $0.327 \pm 0.0106^{3}$ & $0.273 \pm 0.0$ & $0.297 \pm 0.0010^{3}$ & $0.298 \pm 0.0088^{1}$ & 0.2 & 66 & $0.279 \pm 0.0080^{1}$ \\
\hline & $2 b$ & $0.330 \pm 0.0105^{3}$ & $0.333 \pm 0.0078^{3}$ & $0.348 \pm 0.0055^{3}$ & $0.368 \pm 0.0071^{3}$ & $0.323 \pm 0.0065^{3}$ & $0.290 \pm 0.0061^{2}$ & $6 \pm 0.0080$ \\
\hline & $2 \mathrm{c}$ & $333 \pm 0.0100^{3}$ & $0.297 \pm 0.0101^{2}$ & $0.279 \pm 0.0079$ & $0.299 \pm 0$. & 0.2 & $0.290 \pm 0.0038^{3}$ & 109 \\
\hline \multirow{4}{*}{$\begin{array}{c}\text { AWM } 280 \\
\text { nm, } \\
\text { conventional } \\
\text { units }\end{array}$} & 1 & & & & 0 & & & \\
\hline & $2 \mathrm{a}$ & 0 & $8^{3}$ & 0.2 & 0.28 & 0.2 & $0.284 \pm 0.0055^{3}$ & 027 \\
\hline & $2 b$ & $0.316 \pm 0.0078^{3}$ & $0.304 \pm 0.0026^{3}$ & $0.310 \pm 0.0040^{3}$ & $0.317 \pm 0$ & $0.320 \pm 0$ & $0.275 \pm 0.0048^{3}$ & $0.296 \pm 0.0062^{2}$ \\
\hline & $2 \mathrm{c}$ & 0.30 & $0.270 \pm 0.0031^{2}$ & $0.297 \pm 0.0040^{3}$ & $0.292 \pm 0$ & 0.2 & $0.280 \pm 0$ & $0.288 \pm 0.0051^{1}$ \\
\hline \multirow[t]{4}{*}{ SCE, \% } & 1 & & 090 & & $35.0 \pm 1$ & & $33.9 \pm 1$ & $35.0 \pm 1.33$ \\
\hline & $2 \mathrm{a}$ & $5^{3}$ & $9^{2}$ & & & $6^{1}$ & $37.5 \pm$ & $37.9 \pm 1.20$ \\
\hline & $2 b$ & & $41.0 \pm 1.08^{3}$ & $41.4 \pm 1.25^{3}$ & 43.0 & $42.0 \pm$ & $40.0 \pm 1.33^{3}$ & $37.8 \pm 0.93$ \\
\hline & $2 \mathrm{c}$ & $40.8 \pm 1.05^{3}$ & $40.0 \pm 0.92^{2}$ & $39.7 \pm 1.73^{2}$ & $38.0 \pm 1.48$ & $38.0 \pm 0.75^{3}$ & $38.8 \pm 1.17^{2}$ & $37.5 \pm 1.08$ \\
\hline \multirow[t]{4}{*}{$\mathrm{MDA}, \mu \mathrm{M} / \mathrm{L}$} & 1 & $0.60 \pm 0.016$ & $0.55 \pm 0.035$ & $0.58 \pm 0.025$ & $0.54 \pm 0.037$ & 20 & $0.58 \pm 0.421$ & $0.62 \pm 0.038$ \\
\hline & $2 \mathrm{a}$ & $1.65 \pm 0.020^{3}$ & $1.56 \pm 0.040^{3}$ & $1.53 \pm 0.027^{3}$ & $1.37 \pm 0.041^{3}$ & $1.05 \pm 0.050^{3}$ & $0.85 \pm 0.038$ & $0.60 \pm 0.057$ \\
\hline & $2 b$ & $1.64 \pm 0.011^{3}$ & $1.67 \pm 0.038^{3}$ & $1.63 \pm 0.045^{3}$ & $1.62 \pm 0.025^{3}$ & $1.58 \pm 0.033^{3}$ & $1.60 \pm 0.050^{1}$ & $1.53 \pm 0.039^{3}$ \\
\hline & $2 \mathrm{c}$ & $1.63 \pm 0.030^{3}$ & $1.60 \pm 0.017^{3}$ & $1.58 \pm 0.031^{3}$ & $1.58 \pm 0.047^{3}$ & $1.55 \pm 0.040^{3}$ & $1.57 \pm 0.042^{1}$ & $1.52 \pm 0.015^{3}$ \\
\hline
\end{tabular}

Note $-1-p \leq 0.05,2-p \leq 0.01,3-p \leq 0.001$ in comparison with clinically healthy animals (column 1 ).

\section{Discussions}

The research results showed the endogenous intoxication becoming one of the constant components of the pathogenesis of bronchopneumonia of calves. A nonspecific marker of intoxication becomes the level of medium-weight molecules (500-5000 Da) in the blood, and the study on waves of different lengths made possible to clarify the reasons for their increase [10]. Thus, increased AWM values at a wavelength of $237 \mathrm{~nm}$ in patients during the height of the disease and the first day after therapy course completion indicates the predominance of a resorptive mechanism of development of auto-intoxication, in which toxic metabolites are formed and absorbed mainly in natural and artificial cavities of the body, particularly in the lungs.

A decrease in their volume gives reason to note a decrease in the severity of the pathological process in the respiratory tract. An increase in the content of this AWM fraction on the 10th day of convalescence in $41.7 \%$ of patients who have been ill indicates a renewal of the main or development of a secondary disease with damage to the abdominal organ (gastrointestinal tract, genitourinary system).

The leading role in the manifestation of the resorptive mechanism of autointoxication belongs to a relatively high antigenic load, excessive formation of bacterial toxins and microbial waste products [13-15]. The AWM level at a wavelength of $254 \mathrm{~nm}$ mainly increases when the kidneys and liver are involved in the pathological process, and at a wavelength of $280 \mathrm{~nm}$, when metabolic and liver functions are impaired, which indicates the retention and metabolic processes in the mechanism of intoxication development.

The noted situation was observed during the height of the disease in the first days of recovery, but $41.7 \%$ of patients with metabolic disruptions persisted until 13 days. In this mechanism of endogenous intoxication development, the main role belongs to the accumulation of tissue breakdown products, normal or pathological metabolism, which have toxic properties [16].

However, despite the improvement of metabolism, liver and kidney functions, most patients $(62.5 \%)$ retain high activity of free radical oxidation, in particular lipid peroxidation (LPO), which is confirmed by the high level of malondialdehyde. Excess LPO products cause a change in the redox status of pulmonary vascular cells, causing their spasm and the development of local hypoxia [17]. It can disrupt reparative regeneration and increase the severity of residual bronchopneumonia.

The accumulation of toxic metabolites has a negative effect on the erythrocyte membranes [18] which is manifested in an increase of their permeability and transmembrane loss of hemoglobin. It was revealed in $62.5 \%$ of patients during the entire controlled recovery period ( 25 days). In addition, a decrease in the sorption capacity of erythrocyte membranes was indicated by high SCE values.

The degree of "slagging" of erythrocyte membranes decreases first recovery days, but $41.7 \%$ of patients remain having high degree until the $3 \mathrm{~d}$ week of convalescence. Violation of the structure and functions of erythrocyte membranes in bronchopulmonary diseases becomes the reason of secondary hypoxia development 
with a corresponding slowdown in the oxidation and reduction of NADP, a risk of increased severity of respiratory failure, and toxicological consequences of anaerobic metabolism $[19,20]$.

\section{Conclusion}

Study results in a large livestock complex and experiments on clinically healthy calves and patients with bronchopneumonia provided new research on the pathogenesis of respiratory diseases. The height of bronchopneumonia in patients resulted a pronounced syndrome of endogenous intoxication. Their markers remain at a high level for some time after the treatment course completion and the disappearance of clinical symptoms of the underlying disease.

At the same time, the leveling of the individual components of this syndrome does not occur simultaneously. During the first 3 days after completion of the course of treatment, the resorptive markers disappear, and after 1-2 weeks - retention and metabolic auto-toxicity. However, the high activity of free radical oxidation, in particular lipid peroxidation, persisted throughout the study period of recovery ( 25 days).

The membrane structures normalization, particularly red blood cells, occurs at a later date. In this case, their sorption capacity is first restored, after that the process takes places in transmembrane transport. Functional and metabolic changes occurring in the body of calves during the period of convalescence are explained by a high degree of individual variability, but three groups of animals were distinguished by their severity nature. In $37.5 \%$ of those who are ill, the markers of autointoxication disappear within 10-13 days after the treatment course completion.

The AWM level increases in $41.7 \%$ of calves after positive dynamics in 10-13 days at a wavelength of 237 $\mathrm{nm}$. This is an indication of the resorptive mechanism activation of auto-toxicity and the risk of relapse. The dynamics of the recovery processes in $20.8 \%$ of patients with disease is characterized by the normalization of most of the studied parameters, but the preservation of the increased level of lipid peroxidation products and transmembrane hemoglobin loss, which can cause secondary anemia and re-illness.

Endogenous intoxication is a constant component of the pathogenesis of bronchopneumonia in calves and is an integral result of a complex set of pathophysiological phenomena leading to the accumulation of bacterial and metabolic endotoxins in biological media of the body. That have a damaging effect on cellular structures, followed by a cascade of metabolic disorders. The severity of this syndrome determines the severity of the course of bronchopneumonia, the usefulness of the functional and metabolic changes during convalescence and the outcome of the disease.

\section{References}

1. D. Francoz, S. Buczinski, A.M. Bélanger et al., J. Vet. Intern. Med. 29(1), 381-387 (2015)

2. J. Kirchhoff, S. Uhlenbruck, K. Goris, G. Keil, Vet. Res. 45, 20 (2014)

3. J. Bojkovski, D. Milanov, S. Savić et al., Bull. UASVM Veter. Med. 71(2), 313-320 (2014)

4. B. Pardon, J. Alliët, R. Boone et al., Prev. Vet. Med. 120(2), 169-176 (2015)

5. A. Delabouglise, A. James, J-F. Valarcher et al., PLoS One 12(12), e0189090 (2017)

6. Yu.N. Alekhin, M.S. Zhukov, Problems and ways of development of veterinary medicine of high-tech animal husbandry in Proc. of the Int. Sci. and Pract. Conf. dedicated to the 45th anniversary of the GNU "VNIVIPFiT" of the Russ. Agricult. Acad. 31-33 (2015)

7. D.M. Musher, A.R. Thorner, N. Engl. J. Med. 371(17), 1619-1628 (2014)

8. S.J. Deventer, J.W. Cate, G.N. Tytgat, Gastroenterology 94(3), 825-831 (1998)

9. Z.H. Wang, H. Iguchi, G. Ohshio, Pancreas 13, 173-183 (1996)

10. Yu.N. Alekhin, Endogenous intoxication in animals and their diagnosis 10 (Voronezh, 2000)

11. O.A. Tonkoshkurova, A.I. Dmitriev, R.E. Dmitrieva, Clinical laborat. Diagn. 2, 21-22 (1996)

12. M.I. Retskij, S.V. Shabunin, G.N. Bliznetsova et al., Methodical provisions on the study of free radical oxidation processes and the system of antioxidant defense of the body (Voronezh, 2010)

13. X. Wang, P. Quinn, Ser. Subcellular Biochemistry 53, 3-67 (2010)

14. M.Yu. Yakovlev, Human Physiology 29(4), 476-486 (2003)

15. C. Brun-Buisson, E. Roupie, Rev. Pract. 45(14), 1797-1803 (1995)

16. P.H. Andersen, Acta Veterinaria Scandinavica 44(1), 57 (2003)

17. E.K. Weir, S.L. Archer, Respir Physiol. Neurobiol. 174(3), 182-191 (2010)

18. E. Moeendarbary, A.R. Harris, Wiley Interdiscip Rev. Syst. Biol. Med. 6(5), 371-388 (2014)

19. K.I. Proshchaev, N.A. Zabinyakov, K.S. Azarov, P.G. Dovgii, Bull. Exper. Biol. 158(2), 256-259 (2014)

20. T.C. Bartter, M.R. Pratter, W. Abouzgheib et al., Respiratory Failure, Part I: A Physiologic Approach to Respiratory Failure 7rd ed. (Philadelphia, 2011) 